\title{
ON THE CONTINUITY OF BEST POLYNOMIAL APPROXIMATIONS
}

\author{
S. J. POREDA
}

\begin{abstract}
Suppose $f$ is a continuous complex valued function defined on a compact set $E$ in the plane and $p_{n}(f, E)$ is the polynomial of degree $n$ of best uniform approximation to $f$ on $E$. If a polynomial $q_{n}$ of degree $n$ approximates $f$ on $E$ "almost" as well as $p_{n}(f, E)$, then $q_{n}$ is "almost" $p_{n}(f, E)$. Sharp estimates, one for the real and one for the general case, are found for $\left\|q_{n}-p_{n}(f, E)\right\|_{E}$ in terms of the quantity $\left(\left\|f-q_{n}\right\|_{E}-\left\|f-p_{n}(f, E)\right\|_{E}\right)$, where $\|\cdot\|_{E}$ denotes the uniform norm on $E$.
\end{abstract}

1. Introduction. For a function $f$ continuous on $E$, a compact set in the plane, let $\|f\|_{E}=\max _{z \in E}|f(z)|$. Also, for $n \in Z^{+}$, let $p_{n}(f, E)$ denote the polynomial of degree $n$ of best uniform approximation to $f$ on $E$. A basic question that arises in the theory of best approximation is: If two continuous functions $f_{1}$ and $f_{2}$ are "close" on $E$, are their polynomials of best approximation $p_{n}\left(f_{1}, E\right)$ and $p_{n}\left(f_{2}, E\right)$ also "close" on $E$. More precisely, if $\left\{f_{m}\right\}_{m=1}^{\infty}$ is a sequence of continuous functions converging uniformly to $f$ on $E$, does the sequence $\left\{p_{n}\left(f_{m}, E\right)\right\}_{m=1}^{\infty}$ converge uniformly to $p_{n}(f, E)$ on $E$ (for each $n$ ) and if so, how rapid is the convergence.

The above problem can be stated in even greater generality. Suppose $f$ is continuous on a compact set $E, n \in Z^{+}, p_{n}(f, E) \equiv 0,\|f\|_{E}=1$ and $q_{n}$ is a polynomial of degree $n$ for which $\left\|f-q_{n}\right\|_{E} \leqq 1+\varepsilon$, where $\varepsilon>0$. Then, does $\left\|q_{n}\right\|_{E}$ approach zero as $\varepsilon$ approaches zero and if so is there any relationship between their respective rates of convergence to zero. For example, is $\left\|q_{n}\right\|_{E}=O\left(\varepsilon^{\beta}\right)$ for some $\beta>0$ ? We consider the real case first.

2. The real case. Our problem in the real case was settled in 1958 by G. Freud [4] who showed that $\left\|q_{n}\right\|=O(\varepsilon)$ where " $O$ " depends only on $E$ and $f$. His result also holds for approximation by generalized real valued polynomials (cf. Meinardus [1, p. 22]). We shall now state and prove Freud's result for ordinary polynomials and in the process describe

Received by the editors December 2, 1971 and, in revised form, March 2, 1972.

AMS 1969 subject classifications. Primary 4140; Secondary 3070.

Key words and phrases. Best uniform approximation.

(c) American Mathematical Society 1973 
in some way how " $O$ " depends on $E$ and $f$. Our proof shall also serve as a motivation for the corresponding proof in the complex case.

THEOREM 1. Suppose $f$ is continuous and real valued on $E$, a compact subset of the real line, $p_{n}(f, E) \equiv 0,\|f\|_{E}=1$ and $q_{n}$ is a real polynomial of degree $n$ for which $\left\|f-q_{n}\right\|_{E}<1+\varepsilon$ where $\varepsilon>0$. It then follows that $\left\|q_{n}\right\|_{E}=O(\varepsilon)$, where " $O$ " depends only on $E$ and $f$. Furthermore this estimate is sharp for each $n$.

Proof. By Chebyshev's Theorem [1, p. 20] there exist $n+2$ points $\left\{x_{k}\right\}_{k=1}^{n+2}$ in $E$ such that

$$
\begin{aligned}
& x_{1}<x_{2}<\cdots<x_{n+2}, \text { and } f\left(x_{k}\right)=-f\left(x_{k+1}\right) \\
& \text { for } k=1,2, \cdots, n+1 \text {. }
\end{aligned}
$$

We may assume without loss of generality that $f\left(x_{k}\right)=(-1)^{k}, k=$ $1,2, \cdots, n+2$. Let $w(x)=\prod_{k=1}^{n+2}\left(x-x_{k}\right), \quad M=\max _{k}\left|w^{\prime}\left(x_{k}\right)\right|$ and $m=$ $\min _{k}\left|w^{\prime}\left(x_{k}\right)\right|$. We now claim that $\left|q_{n}\left(x_{k}\right)\right|<(n+1) M \varepsilon / m$, for $k=1,2, \cdots$, $n+2$.

If for some $j, 1 \leqq j \leqq n+2,\left|q_{n}\left(x_{j}\right)\right| \geqq(n+1) M \varepsilon / n$, then for some $l \neq j$, $1 \leqq l \leqq n+2,\left|q_{n}\left(x_{l}\right)\right| \geqq \varepsilon$ and

$$
\operatorname{sign}\left[\frac{q_{n}\left(x_{l}\right)}{w^{\prime}\left(x_{l}\right)}\right]=-\operatorname{sign}\left[\frac{q_{n}\left(x_{j}\right)}{w^{\prime}\left(x_{j}\right)}\right] .
$$

This follows, by Lagrange's interpolation formula since,

$$
q_{n}(x)=w(x) \sum_{k=1}^{n+2} \frac{q_{n}\left(x_{k}\right)}{w^{\prime}\left(x_{k}\right)\left(x-x_{k}\right)}=\left(\sum_{k=1}^{n+2} \frac{q_{n}\left(x_{k}\right)}{w^{\prime}\left(x_{k}\right)}\right) x^{n+1}+\cdots,
$$

and so $\sum_{k=1}^{n+2} q_{n}\left(x_{k}\right) / w^{\prime}\left(x_{k}\right)=0$, since $q_{n}$ is a polynomial of degree $n$. Now by the hypothesis of our theorem, $\left|f\left(x_{k}\right)-q_{n}\left(x_{k}\right)\right| \leqq 1+\varepsilon$ for $k=1,2, \cdots$, $n+2$ and so

$$
\begin{aligned}
& \operatorname{sign}\left[q_{n}\left(x_{j}\right)\right]=\operatorname{sign}\left[f\left(x_{j}\right)\right]=\operatorname{sign}\left[(-1)^{j}\right], \quad \text { and similarly, } \\
& \operatorname{sign}\left[q_{n}\left(x_{l}\right)\right]=\operatorname{sign}\left[f\left(x_{l}\right)\right]=\operatorname{sign}\left[(-1)^{l}\right] .
\end{aligned}
$$

If we let, $l=t+j$, then $(-1)^{l}=(-1)^{t}(-1)^{j}$ and so by (2),

$$
\operatorname{sign}\left[q_{n}\left(x_{l}\right)\right]=\operatorname{sign}\left[(-1)^{t} q_{n}\left(x_{j}\right)\right] .
$$

However, $\operatorname{sign}\left[w^{\prime}\left(x_{k}\right)\right]$ alternates on $E$ and, in particular,

$$
\operatorname{sign}\left[w^{\prime}\left(x_{l}\right)\right]=\operatorname{sign}\left[(-1)^{t} w^{\prime}\left(x_{j}\right)\right] .
$$


Thus by (3) and (4) we get

$$
\operatorname{sign}\left[\frac{q_{n}\left(x_{l}\right)}{w^{\prime}\left(x_{l}\right)}\right]=\operatorname{sign}\left[\frac{q_{n}\left(x_{j}\right)}{w^{\prime}\left(x_{j}\right)}\right],
$$

thus contradicting (1). Hence our claim follows.

Now since $E$ is compact, the functions $\left\{w(x) /\left(x-x_{k}\right)\right\}_{k=1}^{n+2}$ are uniformly bounded on $E$, say by $L$, and so again using Lagrange's interpolation formula we can write

$$
\begin{aligned}
\left|q_{n}(x)\right| & =\left|\sum_{k=1}^{n+2} \frac{q_{n}\left(x_{k}\right) w(x)}{w^{\prime}\left(x_{k}\right)\left(x-x_{k}\right)}\right| \leqq \sum_{k=1}^{n+2}\left|\frac{q_{n}\left(x_{k}\right) w(x)}{w^{\prime}\left(x_{k}\right)\left(x-x_{k}\right)}\right| \\
& \leqq \frac{(n+2)(n+1) M L \varepsilon}{m^{2}}, \text { for each } x \in E,
\end{aligned}
$$

where $M$ and $m$ are as before. Hence our theorem follows.

In order to demonstrate that the estimate $\left\|q_{n}\right\|_{E}=O(\varepsilon)$ is sharp for each $n$ let $0 \leqq x_{1}<x_{2}<\cdots<x_{n+2}<1$ and $E=\left\{x_{k}\right\}_{k=1}^{n+2} \cup\{1\}$. Define the function $f$ on $E$ by setting $f\left(x_{k}\right)=(-1)^{k}, k=1, \cdots, n+2$, and $f(1)=0$, and let $q_{n, \varepsilon}(x)=\varepsilon 2^{n}\left(x-\frac{1}{2}\right)^{n}$. Then $f, E$ and $q_{n, \varepsilon}$ satisfy the conditions of Theorem 1; however, $\left\|q_{n, \varepsilon}\right\|_{E}=\left|q_{n, \varepsilon}(1)\right|=\varepsilon$.

\section{The complex case.}

THEOREM 2. Suppose $f$ is continuous on $E$, a compact set in the plane, $n \in Z^{+}, p_{n}(f, E) \equiv 0,\|f\|_{E}=1$ and $q_{n}$ is a polynomial of degree $n$ for which $\left\|f-q_{n}\right\|_{E} \leqq 1+\varepsilon$ where $1>\varepsilon>0$. It then follow's that $\left\|q_{n}\right\|_{E}=O\left(\varepsilon^{\beta}\right)$, for every $\beta<\frac{1}{2}$, where " $O$ " depends only on $E, f$ and $\beta$. Furthermore this estimate is sharp for each $n$ in that it is not in general true for $\beta=\frac{1}{2}$.

Proof. By the Remez condition [3, p. 437] there exists $m$ distinct points $\left\{z_{k}\right\}_{k=1}^{m}$ in $E$ and $m$ positive constants $\left\{\lambda_{k}\right\}_{k=1}^{m}, 2 n+3 \geqq m \geqq n+2$, such that

In particular,

$$
\begin{aligned}
& \text { (i) }\left|f\left(z_{k}\right)\right|=1 \text { for } k=1,2, \cdots, m \text {, and } \\
& \text { (ii) } \sum_{k=1}^{m} \lambda_{k} \overline{f\left(z_{k}\right)} z_{k}^{j}=0, \quad \text { for } j=0,1, \cdots, n \text {. }
\end{aligned}
$$

$$
\sum_{k=1}^{m} \lambda_{k} \overline{f\left(z_{k}\right)} q_{n}\left(z_{k}\right)=0 .
$$

Set $\mu_{k}=f\left(z_{k}\right)$ for $k=1,2, \cdots, m$ and write $q_{n}\left(z_{k}\right)=\left(1+\alpha_{k}\right) \mu_{k}+i \beta_{k} \mu_{k}$, $k=1,2, \cdots, m$, where the $\alpha_{k}$ 's and $\beta_{k}$ 's are real. This can be done in a unique manner. By the hypothesis of our theorem we have that

$$
\left|\alpha_{k} \mu_{k}+i \beta_{k} \mu_{k}\right| \leqq 1+\varepsilon, \text { for } k=1,2, \cdots, m,
$$


so as a consequence

$$
\begin{aligned}
\alpha_{k}^{2}+\beta_{k}^{2} & <(1+\varepsilon)^{2}, \quad \text { and in particular, } \\
\left|\alpha_{k}\right| & <(1+\varepsilon) .
\end{aligned}
$$

With this notation, the expression (5) can be rewritten

or

$$
\sum_{k=1}^{m} \lambda_{k} \bar{\mu}_{k}\left[\left(1+\alpha_{k}\right) \mu_{k}+i \beta_{k} \mu_{k}\right]=0,
$$

$$
\sum_{k=1}^{m} \lambda_{k}+\sum_{k=1}^{m} \lambda_{k} \alpha_{k}+i \sum_{k=1}^{m} \lambda_{k} \beta_{k}=0 .
$$

Equating real parts yields

$$
\sum_{k=1}^{m} \lambda_{k} \alpha_{k}=-\sum_{k=1}^{m} \hat{\lambda}_{k}
$$

We now claim that for any $\beta<\frac{1}{2},\left|q_{n}\left(z_{k}\right)\right|<\varepsilon^{\beta}$, for $k=1,2, \cdots, m$, and all polynomials $q_{n}$ satisfying the conditions of our theorem, if $\varepsilon$ is sufficiently small. If for "sufficiently small" $\varepsilon$ and some $j, 1 \leqq j \leqq m,\left|q_{n}\left(z_{j}\right)\right|>$ $\varepsilon^{\beta}$, it will then follow that

$$
1+\alpha_{j}>\left(\varepsilon^{2 \beta}-2 \varepsilon-\varepsilon^{2}\right) / 2 .
$$

In order to demonstrate this we note that since $\left|q_{n}\left(z_{j}\right)\right|>\varepsilon^{\beta}$, we then have that $\left(1+\alpha_{j}\right)^{2}+\beta_{j}^{2}>\varepsilon^{2 \beta}$, and from (6) we have $(1+\varepsilon)^{2}>\alpha_{j}^{2}+\beta_{j}^{2}$. Combining these two inequalities yields (8).

Now by (7) we have $\sum_{k=1: k \neq j}^{m} \lambda_{k} \alpha_{k}=-\sum_{k=1}^{m} \lambda_{k}-\alpha_{j} \lambda_{j}$, and so

$$
\left|\sum_{k=1 ; k \neq j}^{m} \lambda_{k} \alpha_{k}\right| \geqq \sum_{k=1}^{n} \lambda_{k}+\alpha_{j} \lambda_{j} .
$$

Recalling (6) that $\left|\alpha_{k}\right|<(1+\varepsilon)$ for $k=1,2, \cdots, m$, we obtain

and so

$$
(1+\varepsilon) \sum_{k=1: k \neq j}^{m} \lambda_{k}>\sum_{k=1}^{m} \lambda_{k}+\alpha_{j} \hat{\lambda}_{j},
$$

$$
\varepsilon \sum_{k=1: k \neq j}^{m} \lambda_{k}>\left(1+\alpha_{j}\right) \lambda_{j}>\lambda_{j}\left(\varepsilon^{2 \beta}-2 \varepsilon-\varepsilon^{2}\right) / 2 .
$$

This is impossible if $\varepsilon$ is sufficiently small since $1-2 \beta<0$; hence our claim follows if we note that expression (9) does not depend on $q_{n}$. As in Theorem 1, we can complete our proof and show that $\left\|q_{n}\right\|_{E}=O\left(\varepsilon^{\beta}\right)$ by applying the Lagrange interpolation formula.

In order to demonstrate the sharpness of our result we construct for each $n \in Z^{+}$and each $M>0$ a set $E$ and a function $f$ which satisfy the 
conditions of our theorem and then construct for every sufficiently small $\varepsilon$ a polynomial $q_{n, \varepsilon}(z)$ of degree $n$ for which $\left\|f-q_{n, \varepsilon}\right\|_{E} \leqq 1+\varepsilon$ and such that $\left\|q_{n \cdot \varepsilon}\right\|_{E} \geqq M \varepsilon^{1 / 2}$.

We choose $n+2$ points $\left\{z_{k}\right\}_{k=1}^{n+2}$ such that if $w^{\prime}(z)=\prod_{k=1}^{n+2}\left(z-z_{k}\right)$,

$$
\begin{aligned}
\left|\sum_{k=2}^{n+2} \frac{1}{w^{\prime}\left(z_{k}\right)}\right| & <\sum_{k=2}^{n+2} \frac{1}{\left|w^{\prime}\left(z_{k}\right)\right|}, \text { and let } \\
\tau_{n}(z) & =w(z) \sum_{k=2}^{n+2} \frac{\mu w^{\prime}\left(z_{k}\right)-\left|w^{\prime}\left(z_{k}\right)\right|}{\left|w^{\prime}\left(z_{k}\right)\right| w^{\prime}\left(z_{k}\right)\left(z-z_{k}\right)},
\end{aligned}
$$

where $\mu=\left(\sum_{k=2}^{n+2} 1 / w^{\prime}\left(z_{k}\right)\right) /\left(\sum_{k=2}^{n+2} 1 /\left|w^{\prime}\left(z_{k}\right)\right|\right)$. The polynomial $\tau_{n}$ is not identically constant and so there exists $z_{0}, z_{0} \neq z_{k}, k=1,2, \cdots, n+2$, such that $\left|\tau_{n}\left(z_{0}\right)\right|>M+1$. Let $E=\left\{z_{k}\right\}_{k=0}^{n+2}$ and define a function $f$ on $E$ by setting $f\left(z_{0}\right)=0$ and $f\left(z_{k}\right)=w^{\prime}\left(z_{k}\right) /\left|w^{\prime}\left(z_{k}\right)\right|$ for $k=1,2, \cdots, n+2$. It then follows [2] that $p_{n}(f, E) \equiv 0$ and $\|f\|_{*}=1$.

Now for sufficiently small $\varepsilon$, let $\alpha=a\left(w^{\prime}\left(z_{1}\right) /\left|w^{\prime}\left(z_{1}\right)\right|\right)+i b\left(w^{\prime}\left(z_{1}\right) /\left|w^{\prime}\left(z_{1}\right)\right|\right)$, where $a=-\left(\varepsilon+\varepsilon^{2}\right) / 2$ and $b=\left(\varepsilon-a^{2}\right)^{1 / 2}$. We define $q_{n, \varepsilon}$ by setting $q_{n, \varepsilon}(z)=\left(z-z_{1}\right) q_{n-1}(z)+\alpha$, where $q_{n-1}$ is the polynomial of degree $n-1$ of best uniform approximation to the function $(f(z)-\alpha) /\left(z-z_{1}\right)$ on the set $\left\{z_{k}\right\}_{k=2}^{n+2}$ with respect to the weight function $\left|z-z_{1}\right|$. That is, $q_{n-1}$ minimizes

$$
\max _{2 \leqq k \leqq n+2}\left|z_{k}-z_{1}\right|\left|\left(f\left(z_{k}\right)-\alpha\right) /\left(z_{k}-z_{1}\right)-p_{n-1}\left(z_{k}\right)\right|
$$

for all polynomials $p_{n-1}$ of degree $n-1$. Set

$$
\delta_{n}=\max _{2 \leqq k \leqq n+2}\left|z_{k}-z_{1}\right|\left|\left(f\left(z_{k}\right)-\alpha\right) /\left(z_{k}-z_{1}\right)-q_{n-1}\left(z_{k}\right)\right| .
$$

Note that $\delta_{n}=\max _{2 \leqq k \leqq n+2}\left|f\left(z_{k}\right)-q_{n, \varepsilon}\left(z_{k}\right)\right|$, and so let us first show that $\delta_{n} \leqq 1+\varepsilon$. By applying the work [2] of Motzkin and Walsh, $\delta_{n}$ can be calculated explicitly, in fact

$$
\begin{aligned}
\delta_{n} & =\left|\left(\sum_{k=2}^{n+2} \frac{f\left(z_{k}\right)-\alpha}{w^{\prime}\left(z_{k}\right)}\right) /\left(\sum_{k=2}^{n+2} \frac{1}{\left|w^{\prime}\left(z_{k}\right)\right|}\right)\right| \\
& =\left|1-\alpha\left(\sum_{k=2}^{n+2} \frac{1}{w^{\prime}\left(z_{k}\right)}\right) /\left(\sum_{k=2}^{n+2} \frac{1}{\left|w^{\prime}\left(z_{k}\right)\right|}\right)\right| .
\end{aligned}
$$

Now noting that $\sum_{k=1}^{n+2} 1 / w^{\prime}\left(z_{k}\right)=0$ and by our choice of the $z_{k}$ 's we can write

$$
-\alpha\left(\sum_{k=2}^{n+2} \frac{1}{w^{\prime}\left(z_{k}\right)}\right) /\left(\sum_{k=2}^{n+2} \frac{1}{\left|w^{\prime}\left(z_{k}\right)\right|}\right)=\alpha \sigma\left(\frac{\left|w^{\prime}\left(z_{1}\right)\right|}{w^{\prime}\left(z_{1}\right)}\right)=\sigma a+i \sigma b,
$$

where $1>\sigma \geqq 0$. Thus

$$
\delta_{n}^{2} \leqq(1+\sigma a)^{2}+(\sigma b)^{2}<(1+|a|)^{2}+b^{2}=(1+\varepsilon)^{2} .
$$


By our choice of the value $q_{n, \varepsilon}\left(z_{1}\right)$, a straightforward calculation yields

$$
\left|f\left(z_{1}\right)-q_{n, \varepsilon}\left(z_{1}\right)\right|=1+\varepsilon .
$$

Again by appealing to [2] we can calculate

$$
q_{n-1}(z)=\frac{w(z)}{\left(z-z_{1}\right)}\left[\sum_{k=2}^{n+2} \frac{f\left(z_{k}\right)-\alpha}{w^{\prime}\left(z_{k}\right)\left(z-z_{k}\right)}-A_{0} \sum_{k=2}^{n+2} \frac{1}{\left|w^{\prime}\left(z_{k}\right)\right|\left(z-z_{k}\right)}\right],
$$

where

$$
A_{0}=\left(\sum_{k=2}^{n+2} \frac{f\left(z_{k}\right)-\alpha}{w^{\prime}\left(z_{k}\right)}\right) /\left(\sum_{k=2}^{n+2} \frac{1}{\left|w^{\prime}\left(z_{k}\right)\right|}\right) .
$$

Now by substituting the given values for $f\left(z_{k}\right)$ we obtain $\left(z-z_{1}\right) q_{n-1}(z)=$ $\alpha \tau_{n}(z)$. Hence,

$$
\begin{aligned}
\left\|q_{n, \varepsilon}\right\|_{E} & \geqq\left|q_{n}\left(z_{0}\right)\right|=\left|\left(z_{0}-z_{1}\right) q_{n-1}\left(z_{0}\right)+\alpha\right| \\
& \geqq\left|\alpha \tau_{n}\left(z_{0}\right)\right|-|\alpha| \geqq(M+1)|\alpha|-|\alpha|=M \varepsilon^{1 / 2} .
\end{aligned}
$$

Also, $\left|f\left(z_{0}\right)-q_{n, \varepsilon}\left(z_{0}\right)\right|=\left|q_{n, \varepsilon}\left(z_{0}\right)\right| \leqq 1+\varepsilon$ if $\varepsilon$ is sufficiently small, and so by (11) and (12) we get that $\left\|f-q_{n, \varepsilon}\right\|_{E} \leqq 1+\varepsilon$ and our example is complete.

4. Remark. As a consequence of Theorem 2, if a function $f$ is continuous on a compact set $E$ then for each $\beta<\frac{1}{2}$ and $n \in Z^{+}$, there exists a least constant, $M_{n}$, such that if $q_{n}$ is a polynomial of degree $n$ for which $\left\|f-q_{n}\right\|_{E} \leqq\left\|f-p_{n}(f, E)\right\|_{E}(1+\varepsilon)$, where $0<\varepsilon<1$, then

$$
\left\|p_{n}(f, E)-q_{n}\right\|_{E} \leqq\left\|f-p_{n}(f, E)\right\|_{E} M_{n} \varepsilon^{\beta} .
$$

Whether the sequence $\left\{M_{n}\right\}_{n=0}^{\infty}$ is bounded for each $f$ and $E$ remains open. A similar question can be posed in the real case.

\section{REFERENCES}

1. G. Meinardus, Approximation of functions: Theory and numerical methods, Springer, Berlin, 1964; English transl., Springer Tracts in Natural Philosophy, vol. 13, Springer-Verlag, New York, 1967. MR 31 \#547; MR 36 \#571.

2. T. S. Motzkin and J. L. Walsh, On the derivative of a polynomial and Chebyshev approximation, Proc. Amer. Math. Soc. 4 (1953), 76-87. MR 15, 701.

3. V. I. Smirnov and N. A. Lebedev, Functions of a complex variable, "Nauka", Moscow, 1964; English transl., M.I.T. Press, Cambridge, Mass., 1968. MR 30 \#2152; MR 37 \#5369.

4. G. Freud, Eine Ungleichung für Tschebyscheffische Approximations-polynome, Acta Sci. Math. (Szeged) 19 (1958), 162-164. MR 21 \#251.

Department of Mathematics, Clark University, Worcester, Massachusetts 01620 